\title{
Different neural codes serve long and short-term memory functions in primate Hippocampus and Lateral Prefrontal Cortex during virtual navigation
}

\section{Authors}

Corrigan, B. W. ${ }^{1,2,3}$, Gulli, R. A. ${ }^{4,5}$, Doucet, G. ${ }^{6}$, Roussy, M. ${ }^{1,2,3}$, Luna, R. ${ }^{1,2}$, Sachs, A.J. ${ }^{6}$, Martinez-Trujillo, J. C. ${ }^{1,2,3,7}$

${ }^{1}$ Department of Physiology and Pharmacology, University of Western Ontario, London, ON, Canada

${ }^{2}$ Robarts Research Institute, University of Western Ontario, London, ON, Canada

${ }^{3}$ Brain and Mind Institute, the University of Western Ontario, London, ON, Canada

${ }^{4}$ Zuckerman Mind Brain Behavior Institute, Columbia University, New York, NY, USA

${ }^{5}$ Center for Theoretical Neuroscience, Columbia University, New York, NY, USA

${ }^{6}$ The Ottawa Hospital, University of Ottawa, Ottawa, ON, Canada

${ }^{7}$ Lawson Health Research Institute, London, ON, Canada

\section{Abstract:}

The primate hippocampus (HPC) and lateral prefrontal cortex (LPFC) are two brain structures deemed essential to long- and short-term memory functions respectively. Here we hypothesize that although both structures may encode similar information about the environment, the neural codes mediating neuronal communication in HPC and LPFC have differentially evolved to serve their corresponding memory functions. We used a virtual reality task in which animals navigated through a maze using a joystick and selected one of two targets in the arms of the maze according to a learned context-color rule. We found that neurons and neuronal populations in both regions encode similar information about the different task periods. Moreover, using statistical analyses and linear classifiers, we demonstrated that many HPC neurons concentrate spikes temporally into bursts, whereas most LPFC neurons sparsely distribute spikes over time. When integrating spike rates over short intervals, HPC neuronal ensembles reached maximum decoded information with fewer neurons than LPFC ensembles. We propose that HPC principal cells have evolved intrinsic properties that enable burst firing and temporal summation of synaptic potentials that ultimately facilitates synaptic plasticity and long-term memory formation. On the other hand, LPFC pyramidal cells have intrinsic properties that allow sparsely distributing spikes over time enabling encoding of short-term memories via persistent firing without necessarily triggering rapid changes in the synapses. 


\section{Introduction}

The primate lateral prefrontal cortex (LPFC) and hippocampus (HPC) are two regions that integrate high level sensory information and play an important role in memory function. Lesion studies have shown that the hippocampus plays a fundamental role in long-term memory formation, for which hippocampal microcircuits possess enhanced synaptic plasticity (Bittner, Milstein, Grienberger, Romani, \& Magee, 2017) via long-term potentiation (LTP) and long-term depression (LTD) mechanisms (Raus Balind et al., 2019). On the other hand, the prefrontal cortex, particularly its lateral aspect (LPFC areas 9/46), is known to play a fundamental role in short-term memory encoding (Arnsten, 2013; Spaak, Watanabe, Funahashi, \& Stokes, 2017). Short-term memories (e.g., shortly remembering a telephone number) do not need to be necessarily stored long-term and often vanish after they are no longer needed. Therefore, it is reasonable to assume short-term memories do not necessarily trigger synaptic plasticity. One issue that remains unclear is whether neural codes underlying information processing in primate HPC and LPFC neurons have distinctively evolved to service long and short-term memory encoding respectively.

In the nervous system bursts of action potentials encode and transmit information between neurons, and they can trigger changes in connectivity by inducing synaptic plasticity. A basic mechanism that links trains of action potentials to synaptic plasticity is temporal summation: a compression of synaptic events over time that produces coincidence of postsynaptic potentials and triggers changes in individual synapses (Kandel, Schwartz, Jessell, Siegelbaum, \& Hudspeth, 2012). Neuronal firing patterns that cluster spikes over short time intervals such as bursts can produce temporal summation of postsynaptic potential in target neurons inducing synaptic plasticity (Remy \& Spruston, 2007; Thomas, Watabe, Moody, Makhinson, \& O'Dell, 1998) and can often be found in brain areas such as the HPC associated with long-term memory formation (Bliss \& Collingridge, 1993; Lisman, 1997). However, such firing patterns can also be found in areas of the primate neocortex such as the prefrontal cortex classically associated with encoding short-term memories (Womelsdorf, Ardid, Everling, \& Valiante, 2014). An issue that has not been thoroughly studied in primates is how the ability to fire bursts of action potentials compares in HPC and LPFC neurons during behavior.

In the primate lateral prefrontal cortex (areas 8a and 9/46) spike bursts in layer $\mathrm{V}$ neurons are associated with the onset of selective attention and are synchronized with the phase of beta and gamma frequencies in the anterior cingulate cortex (ACC) (Womelsdorf et al., 2014). During tasks involving short-term memory (also referred to as working memory in the non-human primate literature), most studies in primate LPFC have computed spike rates over at least a second and documented the existence of persistent firing (Leavitt, Pieper, Sachs, \& Martinez-Trujillo, 2017). Using linear classifiers these studies have shown that spike rates computed over at least 400-500 millisecond intervals maximize decoded information (Leavitt et al., 2017; Roussy et al., 2021). This may suggest that neurons in LPFC use a sparser code compared to HPC neurons.

One possibility is that in the HPC and the LPFC the time structure of the spike train is 'tailored' to perform different functions. In the HPC, spikes may be concentrated in bursts to maximize the probability of plastic changes in individual synapses via temporal summation. This will be in line with the primary role of the HPC in long-term memory formation and consolidation (Eichenbaum et al., 2016). In the LPFC spikes may be more distributed over time, a sparse code, which may favour information encoded over a larger population of neurons. In the latter scenario ensembles of neurons can temporarily maintain and broadcast short-term memory signals locally and to other brain areas without necessarily triggering plasticity in individual synapses. The latter would be compatible with the role of the LPFC in encoding short-term memory (Fuster \& Alexander, 1971).

Here, we compare the prevalence of spike train bursts in the HPC and LPFC of macaque monkeys performing a spatial navigation memory task in a virtual environment. We 
found that both structures encode information about the task. However, HPC neurons more often compress informative spikes in bursts relative to the LPFC. Using linear decoders, we demonstrate that it is possible to decode task related information from burst rates in the HPC with an accuracy similar to that of decoders using spike rates. On the other hand, in the LPFC burst decoders performed substantially worse than spike rate decoders. Finally, we demonstrate that small ensembles of HPC and LPFC neurons are sufficient to provide task related information, but HPC neurons do so over shorter time windows and with fewer neurons relative to the LPFC.

\section{Results}

We trained 4 monkeys (Macaca mulatta) on a context object association task in a dynamic virtual environment. We recorded the responses of neurons from the Hippocampus (HPC) of two animals and from the lateral prefrontal cortex (LPFC areas 9/46) in the other two animals. During the task animals navigated through a virtual $X$ maze using a joystick (Fig. 1a,b). Upon arriving at a decision point, where the maze branched out into two arms, two objects appeared at the arms' ends. The animal had to select one of the two objects to obtain a reward. The objects were two colored disks and the target object was defined by two features, the color of the object and the texture of the walls (e.g., for objects red and green: when the texture of the maze walls was wood the target was red, but when the texture of the walls was steel the target was green) (see Fig. 1d, methods, and (Gulli et al., 2020)). Monkeys learned a new association (target color-wall) every day until they became proficient at the task (see example trajectories in Figure 1c). Different color-wall associations were achieved by changing the target colors while leaving the wall cues (contexts) the same across sessions. In a 50-trial performance assessment window, they achieved average performances of: 75.8 (monkey W, HPC), 61.3 (monkey R, HPC), 74.5 (monkey T, LPFC), 84.3 (monkey B, LPFC). The theoretical chance performance in this two-alternative-forced-choice task was $50 \%$.

\section{Prevalence of burst firing in HPC and LPFC single neurons}

We recorded the responses of neurons in the HPC using single electrodes (Fig. 1e) and in the LPFC using microelectrode arrays (Utah arrays) implanted in area 9/46 ventral and dorsal to the principal sulcus (Fig. 1f). Data were spike sorted and single action potential times were extracted and synchronized to the different task events. We measured the firing rates of neurons in both areas by averaging the number of action potentials over the entire task period. We examined the ability of neurons in both areas to fire trains of action potentials during the different task periods. The spikes of HPC neurons frequently occurred within 7 millisecond intervals (example on Fig. 2a, and cyan spikes in c). This was not the case for LPFC neurons that fired spikes more sparsely (example in Fig. $2 b, d$ ). We additionally computed the interspike interval (ISI) distribution of the two example units per trial period and for all periods the HPC unit shows a bias to short ISIs (Fig. 2e) compared to the LPFC cell (Fig. 2f). These observations may suggest that HPC neurons fire bursts of action potentials (ISI $<7 \mathrm{~ms}$ ) more often than LPFC neurons.

In order to get a broad perspective on this, we examined the probability density function for ISIs across all neurons with trough to peak widths of at least $400 \mu \mathrm{s}$ in the two regions (Figure 3a). HPC neurons had a large peak below a bursting threshold of $7 \mathrm{~ms}$ ISIs compared to the LPFC. This also suggests that HPC neurons fire more bursts than the LPFC. To test this hypothesis for all recorded neurons, we measured a burst fraction which quantifies the proportion of ISIs at or below $7 \mathrm{~ms}$ in both areas (Fig. 3b,c). HPC neurons had a higher burst fraction (median $=.092$ ) than LPFC neurons (median $=.027)($ rank sum test, $Z(216,367)=$ $10.45, p<0.05$ ) (Fig. 3c). While this is an effective comparison, we noticed that as firing rate 


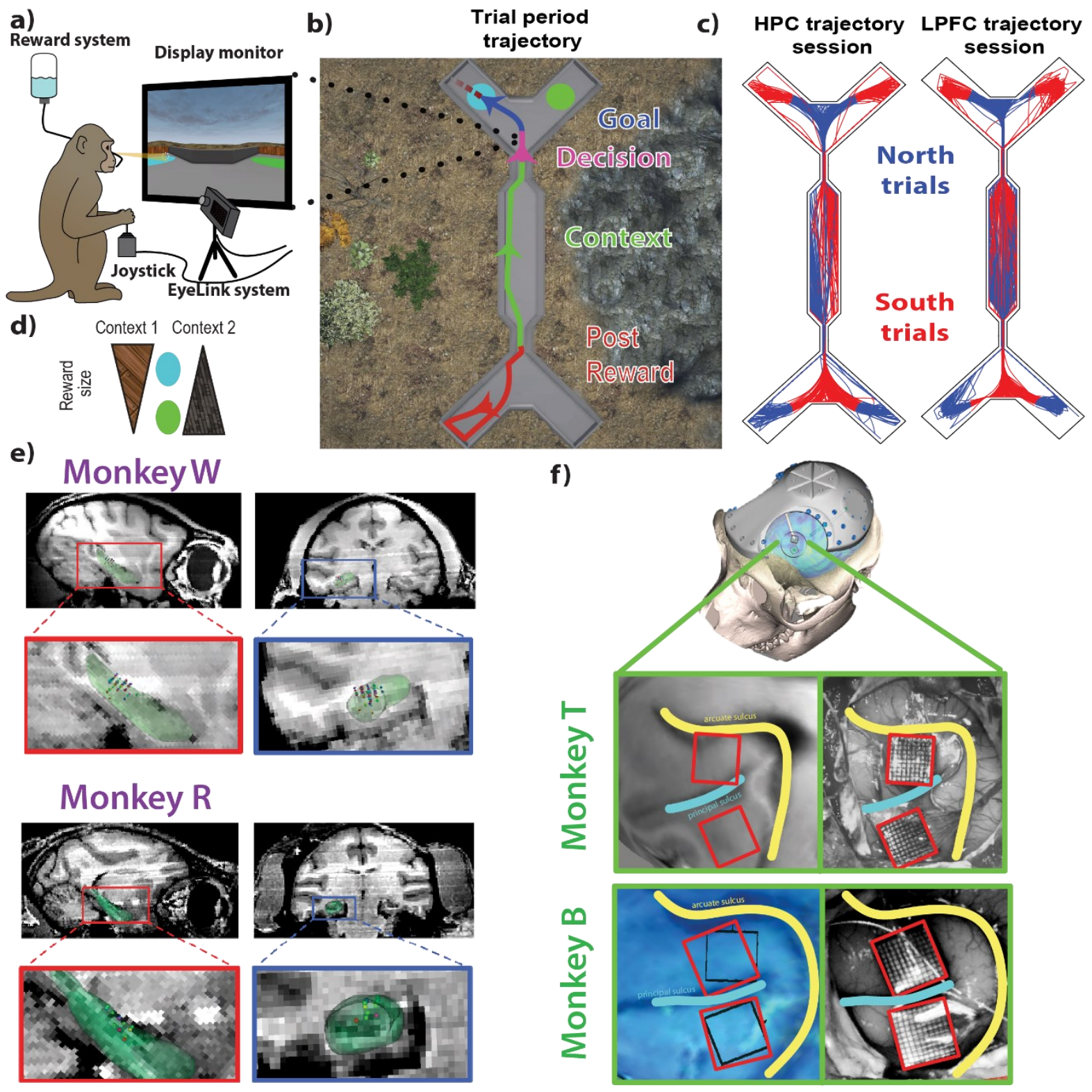

Figure 1. Recording from NHPs during virtual navigation learning task. a) Experimental set-up where monkeys were seated in front of a monitor and used a joystick to navigate the virtual environment. Eye position was monitored, and juice used as reward. b) Top-down view of maze with example trajectory split into four different task periods: Post reward, Context, Decision, Goal (approach). c) Example sessions from monkeys and from each area showing all trajectories separated into north and south trials. d) The rule example defined cyan as the higher value object in context one, and lower in context two, and the inverse for the green object. e) MRI-based reconstruction of recording positions in right hippocampus of NHPs W and R. f) Array locations on area $8 \mathrm{a}$ and $9 / 46$ for NHPs B and T. 
a)

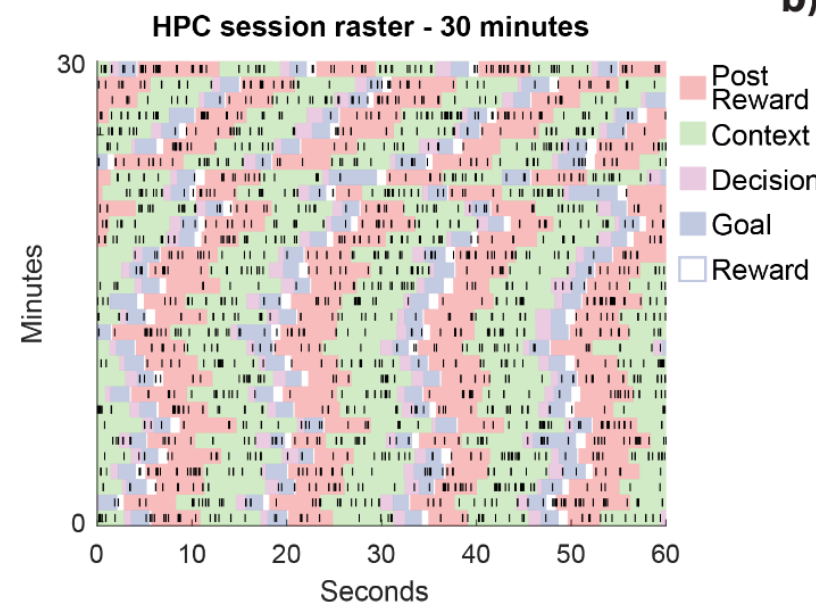

Post Reward trial raster
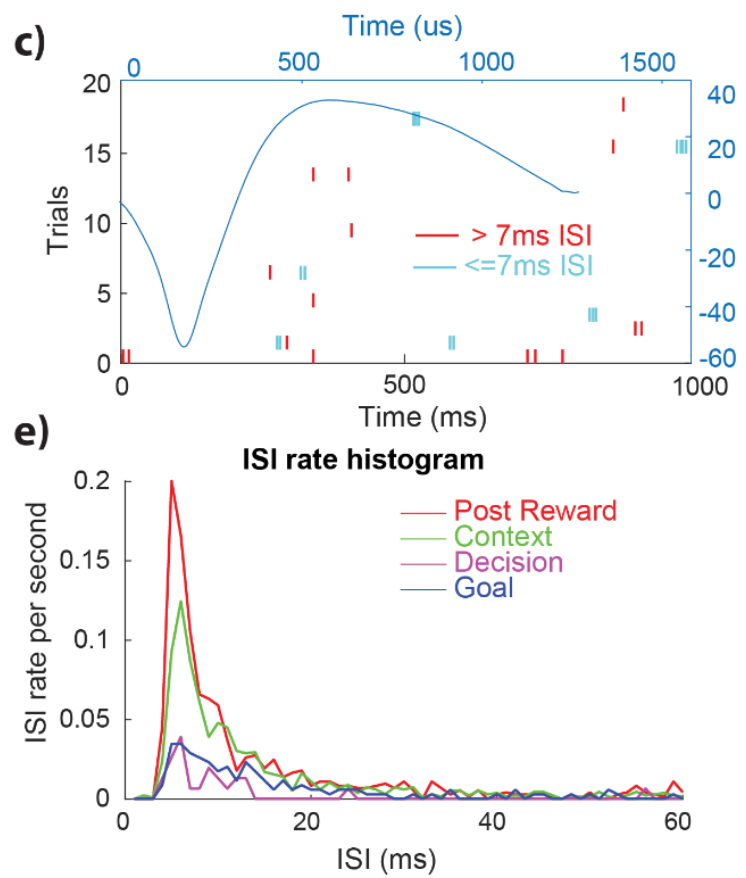

b)

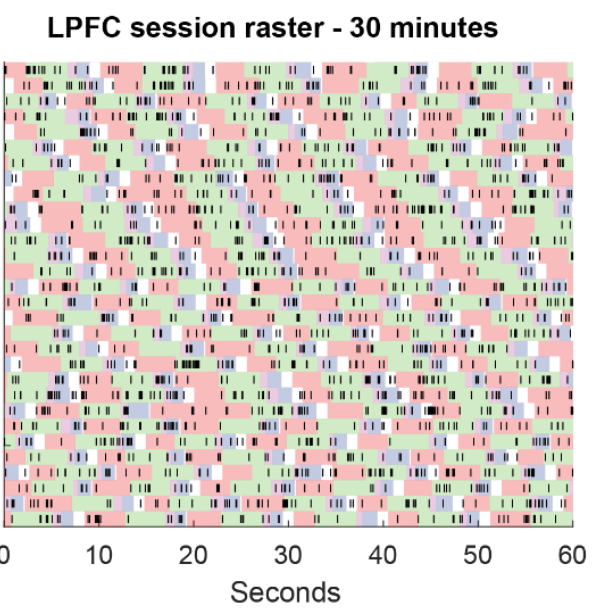

Post Reward trial raster

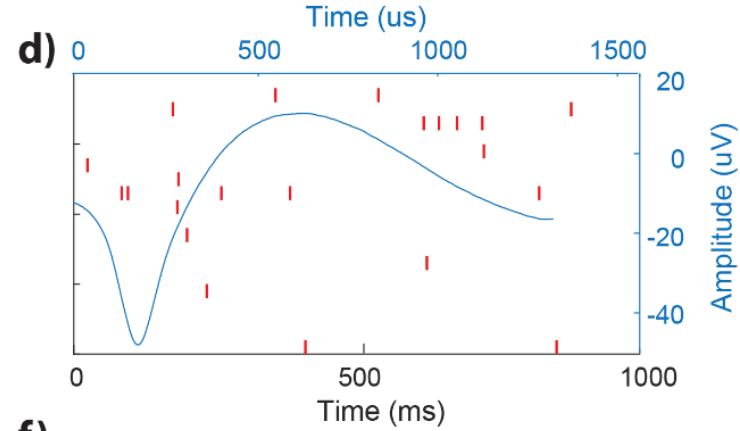

f)

ISI rate histogram

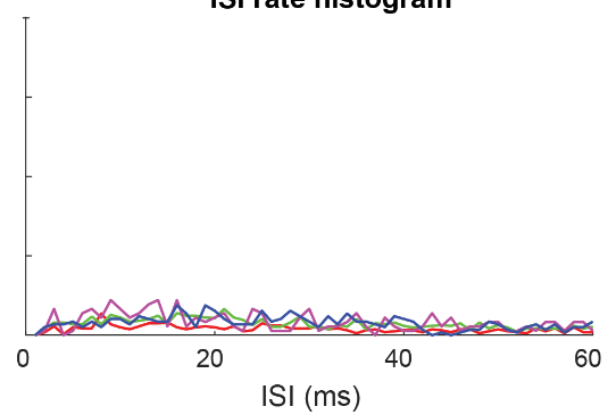

Figure 2. Differences in firing patterns between two example units in $\mathrm{HC}$ and PFC. a,b) Session rasters showing example unit activity for 30 minutes, with background indicating the different trial periods. c,d) Trial rasters for the post reward period with ISIs $<=7$ indicated, occurring more frequently in the hippocampus. Super-imposed are example unit's waveform - broad spiking neurons with similar task firing rate of $1.8 \mathrm{~Hz}$. e,f) Interspike interval (ISI) rates for the different periods. Note that the hippocampal unit has much more activity below $20 \mathrm{~ms}$ than in the following $20 \mathrm{~ms}$, whereas the LPFC unit has a more equitable distribution.

increases, the probability of ISIs being below $7 \mathrm{~ms}$ increases in the LPFC $(r=0.67, p<.05)$. This was not the case in the HPC where the correlation was not statistically significant $(r=-0.06, p>$ .05) (Fig. 3b). The positive correlation between burst fraction and firing rate in LPFC neurons may suggest their ISI distribution is approximated by a Poisson distribution (with some deviance 
as it approaches 0 for the refractory period). The ISI distributions peak close to 0 and then decrease, and this peak rises with the firing rate because the more spikes the neuron fires, the more frequent lower ISIs become while higher ISIs will decrease in frequency.

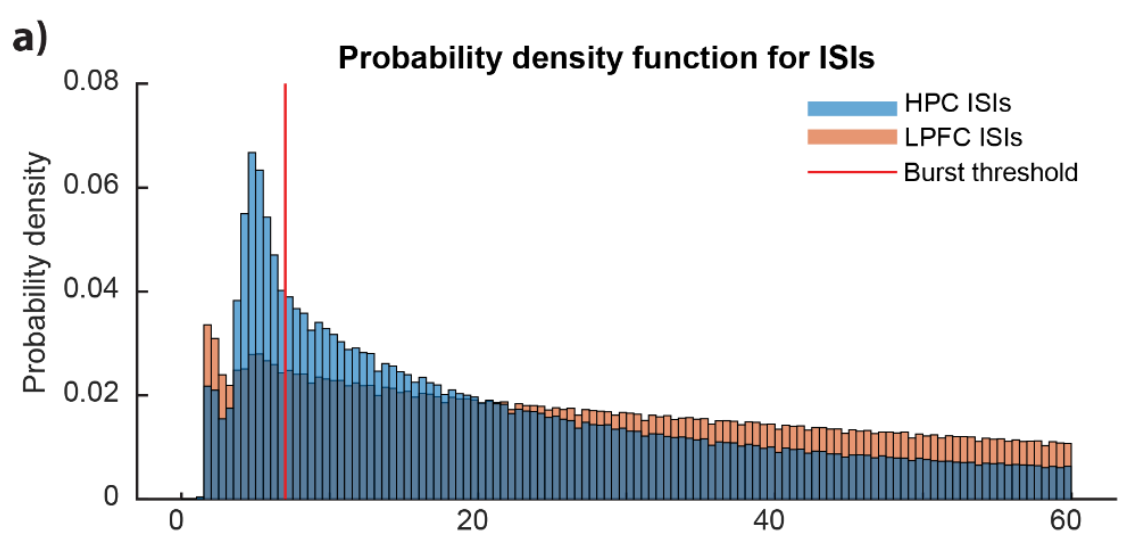

ISI (ms)
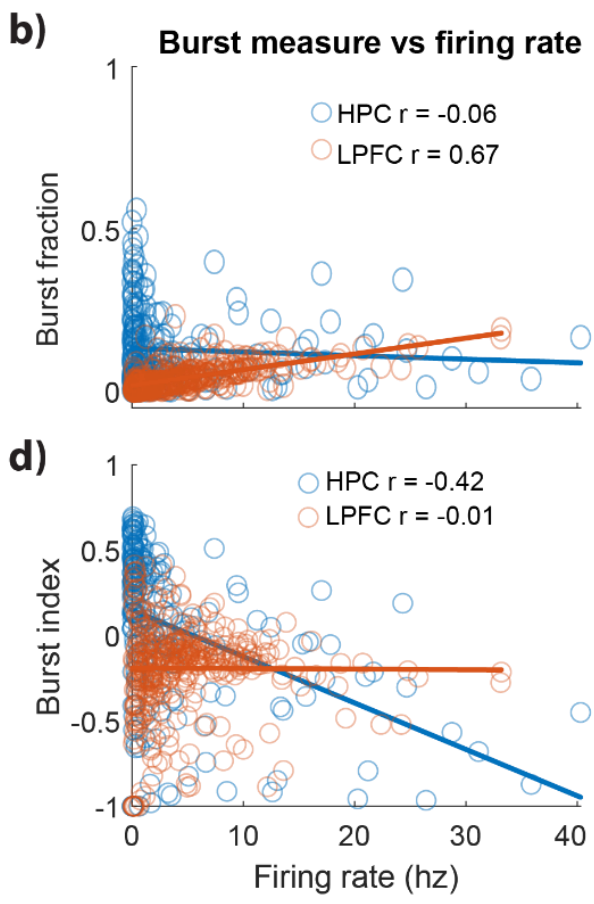

c)
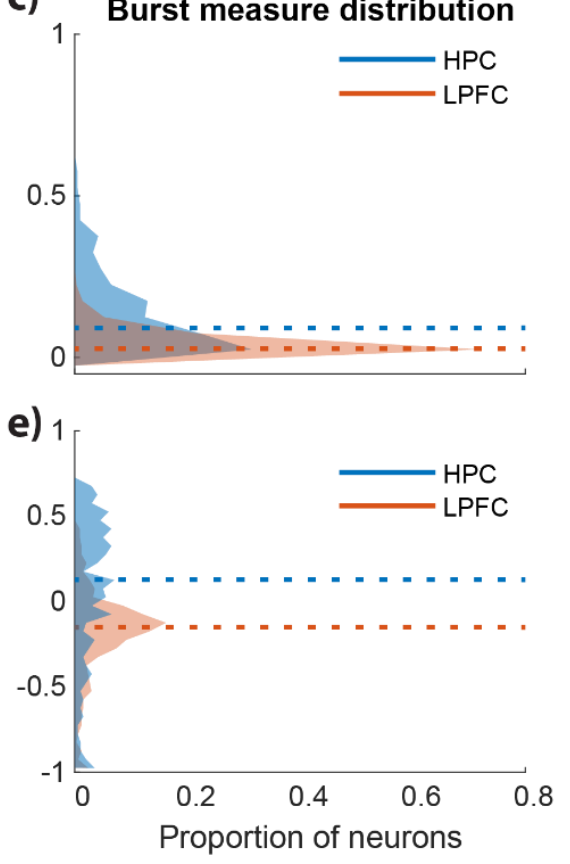

Figure 3. HC cells are more prone to bursting, and PFC burst fraction is correlated with firing rate. a) The probability density distribution of all ISIs across all neurons compared between the two areas. The HPC ISIs have a more pronounced peak below the $7 \mathrm{~ms}$ burst threshold. b) Burst fraction plotted against firing rate, where hippocampal cells have high burst fraction at low firing rates, c) Population density of burst fractions. d) Same as a, but with burst index, where PFC burst index is no longer correlated with firing rate, and there is a slight negative correlation for the $\mathrm{HC}$, see text for equation of burst index. e) Population density of burst indices.

We additionally used a method from Livingstone et al.

(Livingstone, Freeman, \& Hubel, 1996) to compute a probability distribution for ISIs given that a neuron fires in a Poisson fashion. We compared this predicted distribution of ISIs for a neuron with the measured firing rate over the period of the task with the actual distribution to get an index (see methods). This index is a bounded ratio of the measured ISIs and the predicted ISIs. A positive value means there are more short ISIs than predicted by a Poisson distribution, and suggests the cell often fires bursts, while values around or below 0 indicates that the cell does not burst more than predicted for a Poisson firing neuron. We found that the burst index of HPC cells (median $=.14$ ) were significantly higher than the ones of LPFC cells (median $=-.11$, $\mathrm{Z}(216,367)=8.60, p<.05)$ (Fig. 3e). The burst index for LPFC cells did not correlate with firing rate $(r=-0.007, p>.05)$. The burst index of HPC cells was significantly negatively correlated 
with the firing rate $(r=-0.42, p<.05)$ indicating that for higher firing rates HPC cells bursting indices approximate those of Poisson neurons. The latter result may also indicate that at low firing rates many spikes in HPC neurons are concentrated within bursts while at high firing rates neurons fire more spikes 'outside' bursts.

Information about task periods encoded in burst and spike rates in HPC and LPFC Our task had different periods (Post-reward, Context, Decision, Goal Approach and Reward). We examined whether neuronal spikes and/or bursts encoded information about the different task periods in the HPC and LPFC. If a neuron is responding to a specific attribute of the task that allows distinguishing between trial periods, there should be information about trial periods in the responses. An example might be a neuron that responds to one of the target objects presented during the Decision and Goals periods of the task. An alternative might be a neuron that, during the Post-reward period, integrates the reward feedback with the conjunction of the context and the chosen target in the previous trial. A deeper analyses of neuronal selectivities for these task contingencies can be found in Gulli et al. (2020). Here we will first concentrate on overall measurements of information about task periods since our goal is to compare the information contained in burst rates (number of bursts per period) and spikes rates (number of spikes per period) (see methods). This method assumes that if bursts are units of information transmission, then burst rates should encode more or at least similar information as spike rates.

For each neuron we computed mutual information during task periods for spike rates and for burst rates, using a Monte-Carlo test for significance of 5000 shuffles. The spike rate was defined as the firing rate during the entire task period. The burst rate was defined as the number of bursts (at least 3 spikes with ISIs $<7 \mathrm{~ms}$ ) during a task period. To ensure a reasonable sample size we only used neurons with at least 60 completed trials. In monkey $R$ HPC, (Fig. 4a), $62 \%$ of the neurons had significant information corresponding to spike rate, $18 \%$ in the burst rate, and an overlap of $15 \%(n=34)$. In monkey $W, 54 \%$ showed significant information in the spike rate, $22 \%$ in the burst rate, and $20 \%$ for both $(n=186)$. In the LPFC, monkey $\mathrm{T}$ had $55 \%$ of cells with significant mutual information in the spike rate, $10 \%$ in the burst rate, and an overlap of $9 \%(n=129)$. Monkey $B$ had $85 \%$ with spike rate information, while $7 \%$ of cells had burst rate information, all of which also had spike rate information $(n=229)$. Thus, the predominant information was in the spike rates, followed by spike rates + burst rates and finally burst rates alone. These results held for both animals (i.e., pattern of results in the HPC were similar in the two animals, as well as in the LPFC two animals), suggesting that this is not an idiosyncratic pattern present in only one of the animals per area.

Remarkably, burst rate information (burst alone and burst + spike) seems to be more prevalent in HPC than in LPFC neurons. To assess this, we pooled the neurons within the areas and calculated the $95 \%$ confidence intervals for the proportion of cells with bursting information (Fig. 4c). The 95\% confidence interval for the HPC (.16 - .27) did not overlap with the LPFC interval $(.06-.11)$. Thus, there were significantly more cells in the HPC with significant mutual information than in LPFC $(p<.05)$.

In order to quantitatively estimate the amount of information carried in the spike and burst rates of neurons, we plotted rates separately for each monkey (Fig. 4b). For both animals, the information encoded in spike rates was greater than the information encoded in burst rates (Fig. 4b). However, the differences between the information in spikes and bursts seem to be larger in the LPFC than in the HPC. To compare spike and burst rates across areas we pooled neurons from both animals that had recordings in the same regions. We only compared neurons that significantly encoded information either in spike rates or burst rates (permutation test $p<$ .05) (Fig. 4d). In the HPC, spike rate provided significantly more information (median =.14 bits) 
a) Proportion of units with significant mutual information

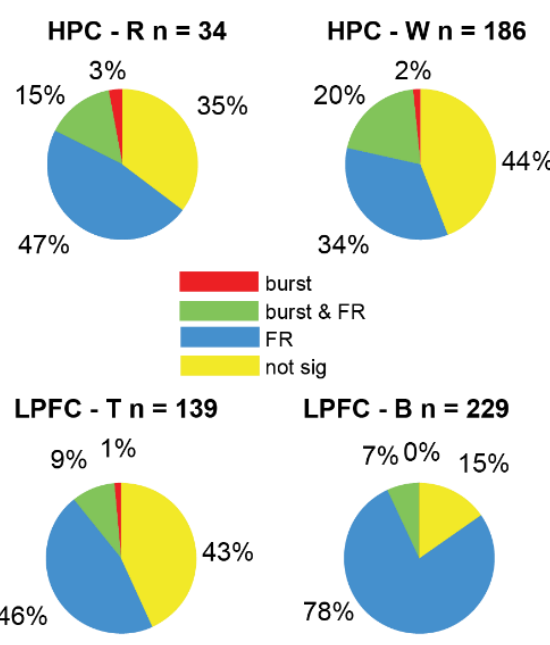

c)

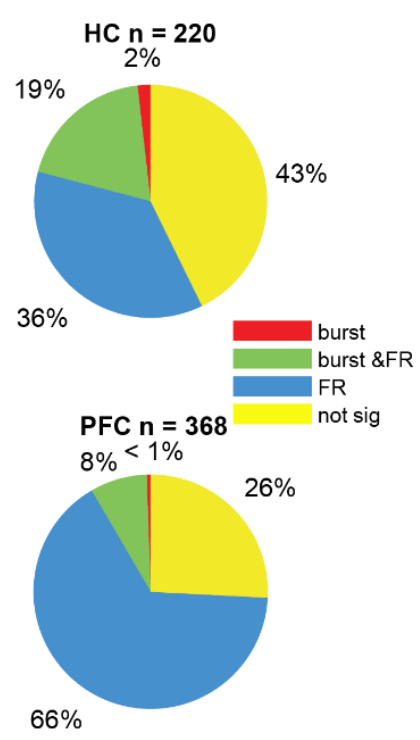

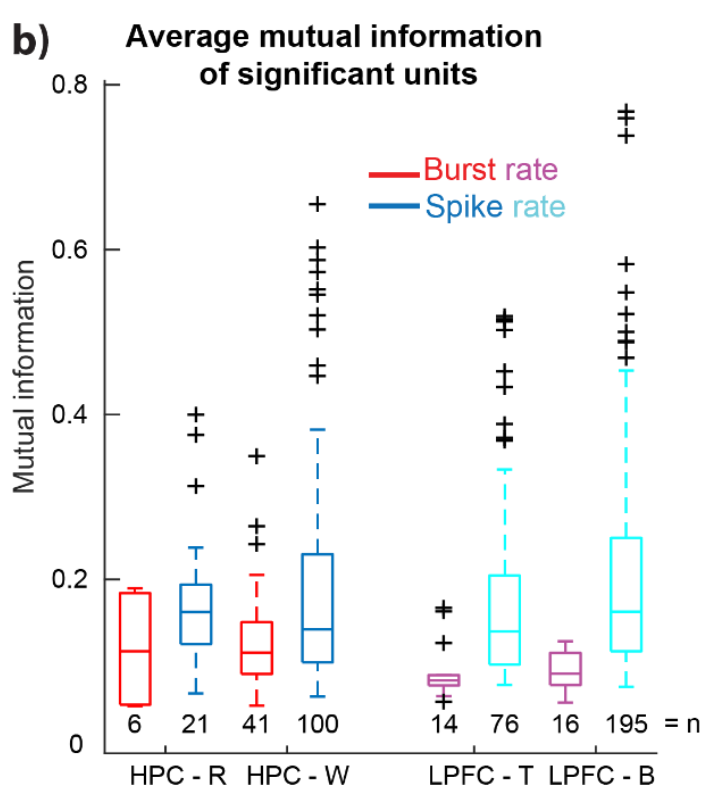

d)

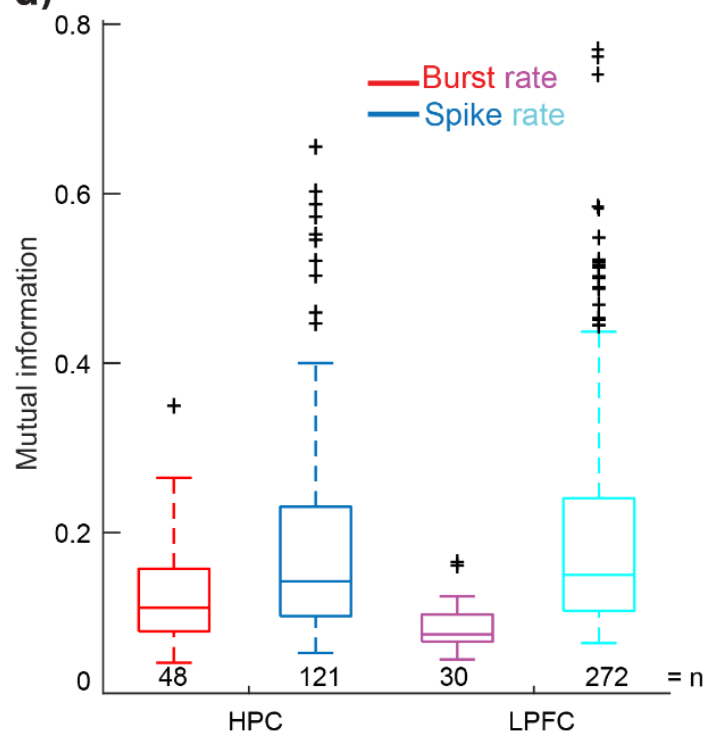

Figure 4. Hippocampal bursts carry more information about the trial period than prefrontal bursts. a) Proportions of units with significant mutual information in bursts, spikes or both. $\sim 1 / 3$ of $\mathrm{HC}$ units with significant spike information have significant burst information. b) Information values for neurons with significant information. c) Proportions of units with significant mutual information for the two areas and across animals. d) Mutual information values for neurons with significant information in (c). Spike rate has more average mutual information per significant neuron in both regions, however bursts have more information in HPC than in LPFC.

than burst rate neurons (median $=.11$ bits, $Z(122,46)=3.13, p<.05$ ). A similar pattern was found in LPFC, where spike rate information (median $=.15$ bits) was significantly higher than the burst rate information (median $=.08$ bits, $\mathrm{Z}(271,31)=6.74, p<.05$ ). There was no significant difference between the information in the spike rate between the HPC and LPFC 
$(Z(271,122)=.72, p>.05)$. However, HPC burst rate cells had significantly more information than the LPFC burst rate cells $(Z(46,31)=-2.92, p<.05)$.

These results show that spike rates in both the HPC and LPFC encode similar information about the different task periods. However, burst rates in the HPC carry more information about task periods than in the LPFC, indicating that HPC neurons compress informative spikes in bursts while LPFC neurons distribute informative spikes more sparsely over time.

\section{Decoding task period information from bursts and spike rates in HPC and LPFC populations}

To determine how results at the level of individual neurons generalize to populations we conducted population level analyses. For the case of the HPC, recordings were done over many days, so we constructed a pseudo population of neurons by pooling neurons and drawing the same numbers of each trial period for each neuron (Mendoza-Halliday \& Martinez-Trujillo, 2017). LPFC data were recorded with microelectrode arrays and contain the original correlation structure of the population. In order to compare LPFC data with HPC data we shuffled the LPFC

a)

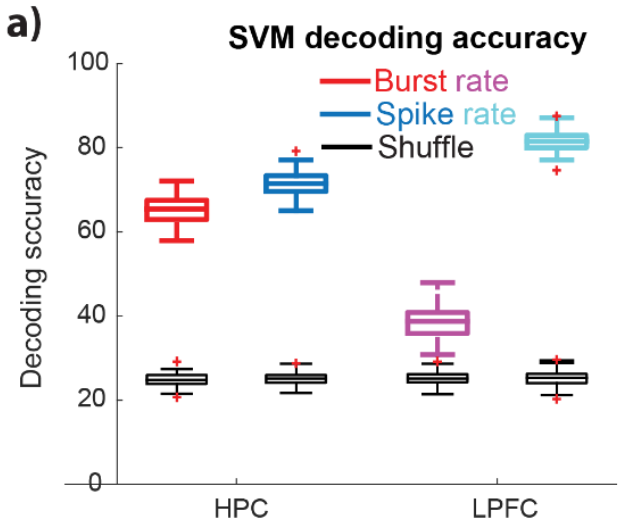

c)

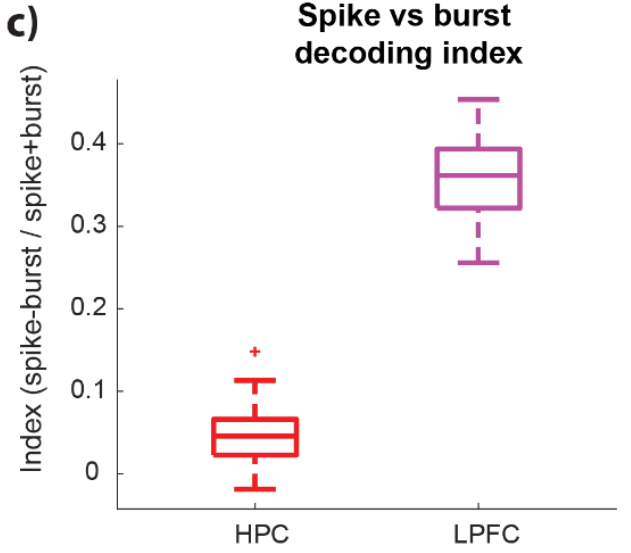

b)

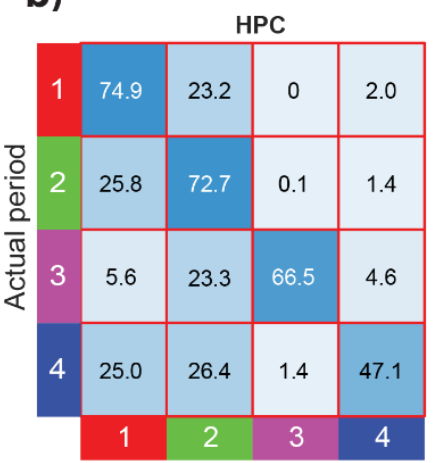

Burst decoding

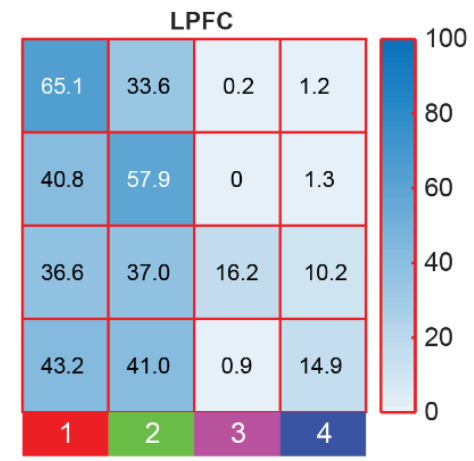

Figure 5. Population code for the hippocampus is similar between bursts and spikes, but not for LPFC. a) Average decoding accuracy for trial period in spikes and bursts codes in the two areas. All values are significantly different from the shuffles. b) Confusion matrices for burst and spike decoding in both areas. There is no distinct diagonal in LPFC burst decoding, but there is in the other three matrices. c) Spike-burst decoding index, where the difference between spike and burst decoding is significantly higher for LPFC, and close to 0 for HPC. 
trial order destroying simultaneity and the correlation structure of the population. Thus, the analyses use pseudo populations of HPC and LPFC neurons. We trained a support vector machine (SVM) to decode the trial period based on the spike rate or the burst rate of a pseudopopulation of 220 cells from each area. We selected 60 trials of each period and used the spike rates and burst rates of these periods as inputs for our decoders. We ran 5-fold cross validation, getting one average performance, and then ran this 100 times to get 100 average performances. We also shuffled trial labels for each population to get random performances and compute chance level decoding ( 25\%).

All decoding performances were significantly different from the chance shuffle with $p<<$ .05 (Fig. 5a). When we analysed performance rates within regions, we found that there was a significant difference between the HPC burst decoding (mean $=65.30 \%$ ) and spike decoding (mean $=71.42 \%$ ). This was not as large as the difference we found in the LPFC between burst $($ mean $=38.65 \%)$ and spike decoding (mean $=82.12 \%)$. To corroborate this observation, we created a spike-burst index: (spike rate performance - burst rate performance) / (spike rate performance + burst rate performance) (Fig. 5c). There was a significant difference between the HPC index (mean $=.045,8 \%$ higher performance for spike rates than burst rates) and the LPFC index (mean $=.36,112 \%$ higher performance for spikes rates than for burst rates) (t-test, $p<$ .05). The difference in the LPFC was more than 10-fold higher than in the HPC. This suggests that burst codes were much more prominent in the HPC than in the LPFC.

Interestingly, the distribution of index values also included negative values for the HPC, showing that in the HPC, decoding from bursts in some instances outperformed decoding from spikes.

One question concerning ensemble coding of task periods from burst and spike rates is whether information encoded is distributed over many neurons or over few neurons only. In order to clarify this issue, we examined how decoded information from both burst and spike rates changes as a function of neuronal ensemble size and composition. For these analyses we used the units that had significant mutual information in their burst rates. Using a procedure similar to Leavitt et al. (2017), we first estimated decoded information in individual neurons, and then we paired the best neurons with every other neuron to find the best duo, grouping that duo with every other neuron to find the best trio etc. (see (Backen, Treue, \& Martinez-Trujillo, 2018; Leavitt et al., 2017)). We ran this process 50 times to generate a population we could further test. For both the HPC and the LPFC, ensembles of a relatively small number of neurons seem to saturate the decoder's performance (Fig. 6a). To quantify this, we fit an exponential function to the data (eq. 4). All fits had an $r^{2}>.95$. We estimated the point at $95 \%$ of the maximum performance in order to conduct comparisons between areas and burst and spike rate decoders. The HPC reached the $95 \%$ rate for spike rates at an average of $5.4(\mathrm{SD}=.9)$ neurons, and for the burst rate at $8.6(S D=2.8)$ neurons, which was significantly higher $(t(98)=$ $5.9, p<.05)$. For the LPFC, firing rate ensembles reached the $95 \%$ point at an average of 3.6 $(\mathrm{SD}=.6)$ units, and burst rate ensembles at $8.8(\mathrm{SD}=2.6)$ neurons, which was also significantly higher than the firing rate $(t(98)=12.9, p<.05)$. The $95 \%$ point was significantly lower for the LPFC than the HPC for firing rate decoders $(t(98)=11.9, p<.05)$, but there was no significant difference between the $95 \%$ points for the burst rates between areas $(t(98)=0.4, p>.05)$. Thus, ensembles with small numbers of neurons $(<10)$ were sufficient to saturate the decoder's performance. Moreover, spike rate decoders that use the full task period time to integrate the rates usually needed smaller ensemble sizes than burst rate decoders.

In order to quantify the differences in performance between burst rate decoders and spike rate decoders in each area we used asymptotes of the performance equation for all of the 50 optimized decoders. We subtracted the burst rate performance from the spike rate performance and divided by the sum of the performances. The HPC performance index was lower (mean of .16 (SD = .04)) than the LPFC index (mean of .36 (SD = .03); $t(98)=29.7, p<$ $.05)$. These results indicate that burst rate contributes significantly more to information decoded from neuronal populations in the HPC than in the LPFC. Importantly, in both structures, 
a)
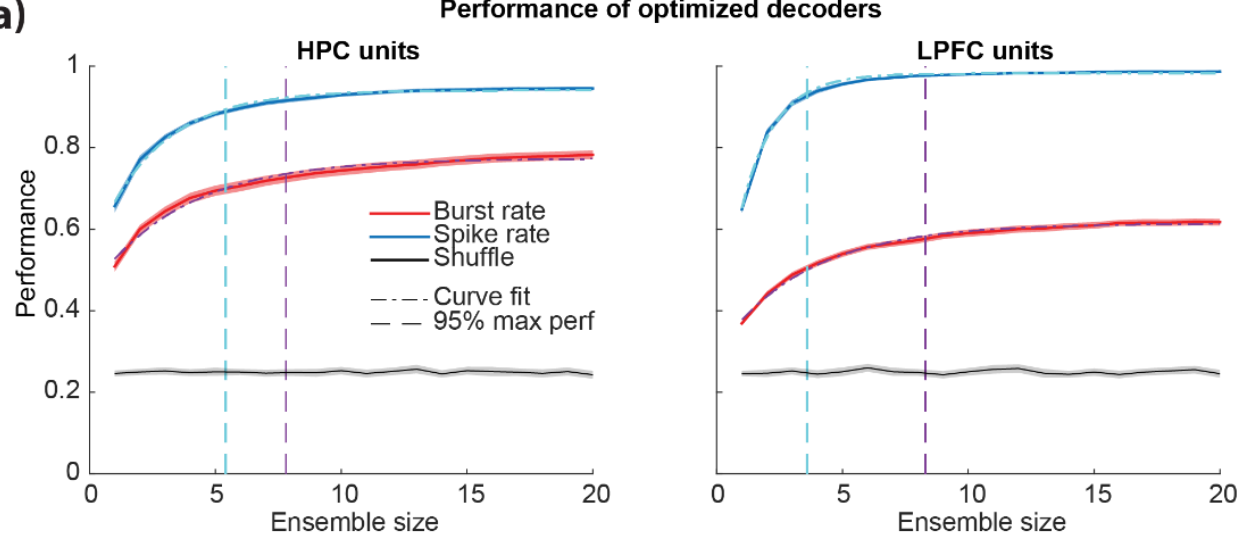

b)
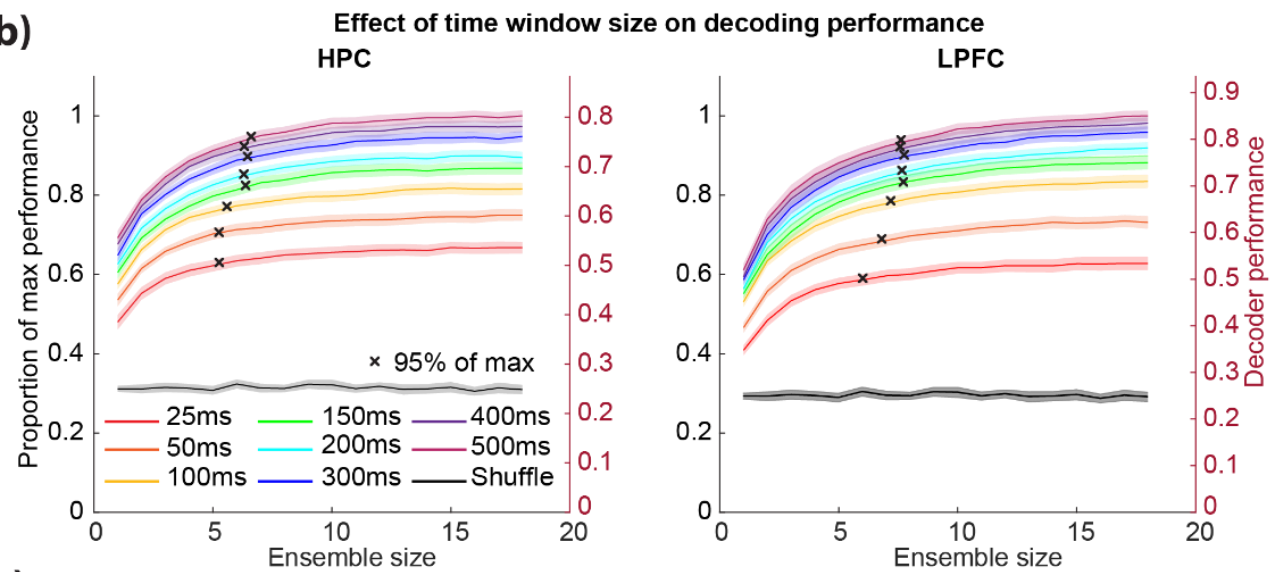

c)

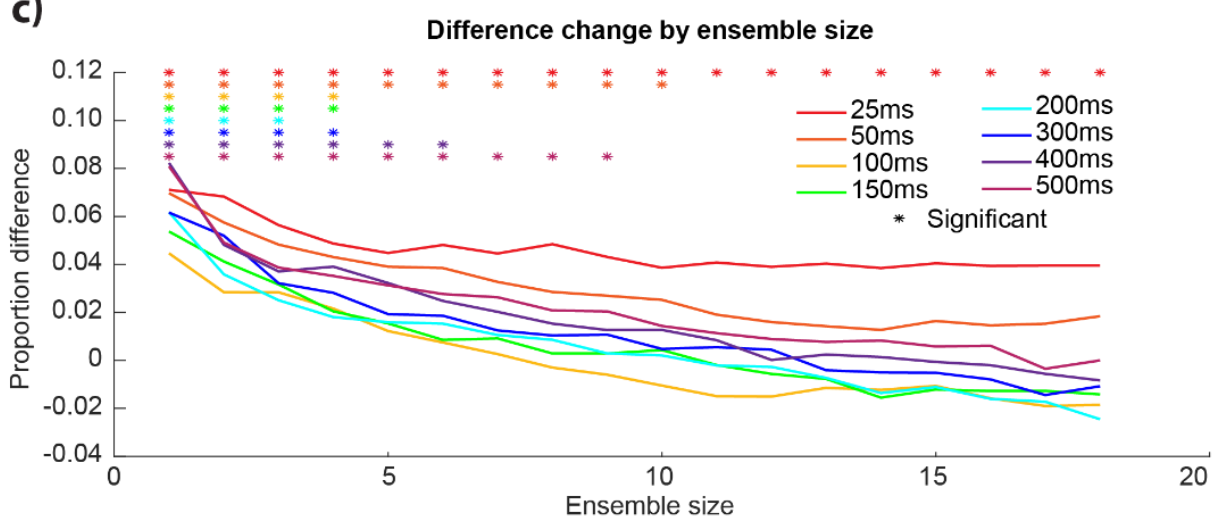

Figure 6. Hippocampal firing rates carry information in compressed time windows and with fewer neurons. a) Optimized ensembles for burst and spike rates yielded by neurons that have significant burst information and their fits to $y=1-\mathrm{a}^{*} \mathrm{e}^{\wedge}(-(\mathrm{x} / \mathrm{b}))-\mathrm{c}$. HPC burst rate ensembles are closer to the performance of HPC spike rate ensembles in terms of maximum performance and in terms of position of the $95 \%$ threshold crossing. b) Optimized decoders for spike rate in different time windows, where HPC ensembles have a smaller range. Left axis is performance as a proportion of maximum achieved performance and right axis is the raw performance. $95 \%$ of maximum point is indicated with an ' $x$ ' on each curve. c) HPC proportion of maximum performance minus LPFC proportion of maximum performance. The difference shows that for HPC, smaller ensembles perform better, as well as performing better in smaller time windows. Asterisks indicate significant difference from $0(p<.05)$. 
available information can be decoded from small ensembles of neurons suggesting that such small subpopulations are sufficient to encode period information during our virtual navigation task.

\section{Effect of integration time windows in decoded information}

The previous results show that neurons in the different structures distribute spikes over different time scales. To study the exact time scales of integration more closely, we examined decoding performance as a function of length of integration time window for both the HPC and the LPFC. The optimized ensemble building technique was applied to the firing rates of neurons that had significant spike rate information. However, instead of integrating over the whole task period, we took different sized time windows centered at the middle of the period (i.e., 25, 50, 100, 150, $200,300,400,500 \mathrm{~ms})$. We repeated the same curve fitting procedure as in figure $6 \mathrm{a}$ and calculated the $95 \%$ point for all the average curves (Fig. 6b). Importantly, the curves in each area were normalized to the maximum asymptotic performance, which happened to be at the longest window (500ms).

We found that in general the 95\% points for the HPC (mean $=6.0, \mathrm{SD}=0.56$ ) were significantly lower than for the LPFC (mean $=7.3(S D=.61)$; paired t-test, $t(7)=13.2, p<.05$ ) (Fig. 6b). This indicates that as a general rule for the different time windows length, the HPC seems to use fewer neurons than the LPFC. Thus, the HPC also compresses spikes across units. We further calculated the performance of each ensemble as a fraction of the maximum of the fit of the curve for the average data in the $500 \mathrm{~ms}$ ensembles. The latter allows subtracting LPFC fractions from the HPC fractions for every time window and ensemble size (HPC-LPFC). A number higher than zero means the HPC needs fewer neurons than the LPFC for achieving a certain proportion of the maximum decoding performance. A negative number means the opposite.

To determine whether ensemble size and time window affect decoding differently in the two areas we conducted a 2-way ANOVA, which was significant for both factors, time window size $(F(7)=41.29, p<.05)$ and ensemble size $(F(17)=28.24, p<.05)$; the interaction was not significant $(F(119)=0.29, p>.05)$. We further carried out two-sided t-tests to determine which differences between areas were significantly different from 0 , indicating that an ensemble of size $\mathrm{n}$ at a particular timepoint performed better in one area than the other $(p<.05)$. As

demonstrated in figure $6 \mathrm{c}$, at lower ensemble sizes across all time windows the HPC ensembles outperform the LPFC ensembles. Remarkably, for the $25 \mathrm{~ms}$ time window, the HPC continues to outperform the LPFC for all ensemble sizes. These results demonstrate that the HPC compresses more of that information into smaller windows of time (25ms) and into fewer units (up to 3) as compared to the LPFC.

\section{Discussion}

We tested four macaque monkeys during a virtual navigation task and recorded neuronal activity from the HPC and the LPFC. We found that although neurons in both areas encoded similar information about the task periods, HPC neurons fired bursts (ISI $<7 \mathrm{~ms}$ ) of informative spikes more often than LPFC neurons. Using linear decoders, we also show that the HPC neuronal ensembles compress informative spikes in time (over windows as short as $25 \mathrm{~ms}$ ) and using fewer neurons relative to the LPFC. In the HPC, but not in the LPFC, a decoder that uses burst rates approximates the performance of a decoder that uses spike rates. Thus, the neural codes in the HPC and LPFC carry similar task related information, but they differ in the way they compress information over time and number of neurons. These differences may result from the different roles of these two structures in long- and short-term memory respectively. 


\section{Burst firing in HPC and LPFC neurons}

The time compression of spike trains in bursts has been previously studied in different model systems (Zeldenrust, Wadman, \& Englitz, 2018). Here we have used the definition of bursts as trains of action potentials that occur with close temporal proximity $(<7 \mathrm{~ms})$ or are more concentrated in time than predicted by a Poisson process, followed by a period of relative quiescence. Using these criteria, we found that the HPC has a larger proportion of putative principal neurons firing bursts than the LPFC. This is in line with previous studies in the HPC that have reported an abundance of burst firing in HPC principal cells across species (Bliss \& Collingridge, 1993; Lisman, 1997; Skaggs et al., 2007; Xu et al., 2012). It also agrees with studies in the LPFC reporting that information decoded during short-term memory and attention tasks is maximized when using time windows of $400 \mathrm{~ms}$ or longer (Backen et al., 2018; Leavitt et al., 2017; Tremblay, Pieper, Sachs, \& Martinez-Trujillo, 2015). However, we should make clear that in both structures, rate codes that count all the spikes inside and outside bursts were more informative than codes counting the number of bursts (burst codes). The latter suggest that not all informative spikes even in the HPC undergo temporal compression in bursts, but they can exist outside the burst. Thus, the function of bursts may not be information coding per se but may be related to other functions such as triggering rapid and effective synaptic plasticity (Zeldenrust et al., 2018).

Our results are unlikely due to differences in the information encoded by the HPC and LPFC. We designed a task in which we could document neurons in both areas encode information about the different task periods (see Fig. 4,5). Previous studies have shown that both the HPC (Doucet, Gulli, Corrigan, Duong, \& Martinez-Trujillo, 2020; Gulli et al., 2020) and the LPFC (Roussy et al., 2021) encode spatial information during navigation tasks. Indeed, our data show that single neurons and neuronal populations in both areas similarly encode task period information. One may also argue that the differences in the number of neurons firing bursts between the two areas may be due to differences in the pattern of eye movements that may have been different between the HPC and the LPFC animals. For example, one may here consider that in both areas saccades are preceded by a presaccadic burst and more saccades may have led to more bursts. In our naturalistic task, where animals could freely make saccades, we found that the durations of intersaccadic intervals, or foveations is similar arguing against the explanation that saccade patterns could be the cause of the differences in burst firing (Supplementary Figure 1). It may also be possible that cells in the different areas encode saccade parameters linked to burst firing distinctively. However, a recent study has reported that during a naturalistic task only $2-3 \%$ of LPFC neurons are tuned for saccade parameters (Roussy et al., 2021). In the HPC, saccades produce phase resetting of LFPs, but HPC neurons are in general not tuned for saccade parameters (Doucet et al., 2020; Gulli et al., 2020).

It has been reported that a feature of HPC principal cells is they frequently fire in very rapid bursts, measured by ISIs below 20ms (Lisman, 1997; Skaggs et al., 2007), or even 6-8ms (Buzsáki, 2015; Ranck, 1973). This physiological burst can make up a significant proportion of all spikes, and a high burst fraction (fraction of all ISIs that are below 20ms) is a distinguishing feature of these HPC cells in primates (Skaggs et al., 2007) as well as rodents (Lisman, 1997). The hippocampal spike burst has consistently proven intriguing to scientists (Kepecs, Wang, \& Lisman, 2002; Lisman, 1997; Zeldenrust et al., 2018). For example bursts with short ISIs <6$8 \mathrm{~ms}$ are quite common along the perforant pathway, from the dentate gyrus to the subiculum regions (Mizuseki, Royer, Diba, \& Buzsáki, 2012; Pernía-Andrade \& Jonas, 2014; Simonnet \& Brecht, 2019). It is currently a matter of debate whether bursts can be the units of information encoded in the HPC (Harris, Hirase, Leinekugel, Henze, \& Buzsáki, 2001). Our results in the HPC suggest that there is information carried by spikes outside the bursts. We could decode task period information from burst rates with a performance much higher than chance. However, when analyzing information decoded from bursts and spike rates (counting spikes within and 
outside the burst), the latter outperformed the former suggesting that spikes outside a burst add to information coding. Importantly, in the LPFC burst decoding was much lower than spike rate decoding suggesting than spikes outside a burst play a larger role in information encoding relative to the HPC.

In the macaque LPFC, few studies have examined the role of burst firing in the coding of working memory signals (Constantinidis \& Goldman-Rakic, 2002; Voloh \& Womelsdorf, 2018; Womelsdorf et al., 2014). Constantinidis and Goldman-Rakic used an unbounded burst index (comparing ISIs with predictions from a Poisson process) in LPFC neurons, and found the median to be .9, just below 1 which would match the Poisson prediction. They calculated their index during the fixation period of an oculomotor delayed response task, so the visual stimulation and mental state were likely quite different from the monkeys in a task like ours, with a dynamic virtual environment. This could explain why they found $10 \%$ of cells with an index value $\geq 4$, which would be approximately . 6 in our bursting index, but we found no LPFC neurons above .5. We also used a slightly larger window of ISIs, so this may have affected our results if ISI peaks drastically dropped off before $7 \mathrm{~ms}$. Other work by the Womelsdorf group in a similar cued attention task found that ISIs $\leq 5 \mathrm{~ms}$ increased during selective attention in LPFC (Womelsdorf et al., 2014). They also found that narrow waveform putative interneuron bursts were synchronized to different LFP phases during attention. These results are not necessarily in contradiction with ours; we did find neurons firing bursts in the LPFC. However, the majority of the informative spikes in LPFC neurons we recorded from occurred outside the burst. Moreover, bursts were not informative when bursts codes were used to decode information about task period, at least compared to the HPC. Overall, our results indicate that bursts are less prominent and play a lesser role in information coding in the LPFC compared to the HPC.

\section{Memory functions of HPC and LPFC and relationship to bursts}

A main hypothesis in our study was that the features of the neural codes in the HPC and LPFC have evolved to serve the functions of these two structures. We found that bursts are more prevalent in the HPC. Given the relationship between burst firing, temporal summation and synaptic plasticity, our results indicate the prevalence of burst firing in the HPC serves the function of this structure in the formation of long-term memories. Indeed, bursting has been shown to be more reliable than single spikes at producing postsynaptic potentials (Remy \& Spruston, 2007; Thomas et al., 1998). Xu and colleagues (Xu et al., 2012) ablated excitatory post-synaptic potentials triggered by single spikes outside bursts by knocking down synaptotagmin-1 in rodent HPC CA1. Their manipulation preserved the potentials triggered by burst spikes. Under these circumstances, learning was preserved. However, when they repeated the manipulation in the medial prefrontal cortex, learning was impaired. Although these experiments were conducted in rodents, their results suggest that in the HPC, burst spikes play a fundamental role in memory formation while spikes outside the burst play a lesser role.

In the LPFC, spike rates integrated over long time intervals ( $>400 \mathrm{~ms}$ ) provide the most information about the items held in short-term memory (Leavitt et al., 2017; Roussy et al., 2021). We reasoned that this sparser code relative to the HPC may avoid triggering synaptic plasticity that would lead to the consolidation of short-term memories as long-term. This would be counter-productive since most short-term memories do not need to be stored long term. It would be wasteful for the brain to trigger long-term memory storage mechanisms every time a shortterm memory is 'loaded' in the LPFC microcircuits. Nevertheless, we did find bursting neurons in the LPFC. These neurons may serve other roles not necessarily related to long-term memory formation, such as broadcasting and communication with other brain areas (Womelsdorf et al., 2014). 
Two distinct architectures for two different memory functions

Differences in the neural codes employed by the HPC and LPFC may be due to different cortical architectures that have evolved to serve different functions. Indeed, there are anatomical differences between the Hippocampus and the prefrontal cortex. The Hippocampus contains three cortical layers (paleocortex) and a relatively well-mapped circuitry in terms of input and output pathways (O'Keefe \& Nadel, 1978). The HPC CA subfields have limited connectivity with areas of the neocortex, but the principal cells' axons follow a well-defined connectivity pattern that ends in the subiculum. Output from the HPC is almost exclusively through the subiculum and the entorhinal cortex. On the other hand, The LPFC is extensively connected to many other brain structures (Yeterian, Pandya, Tomaiuolo, \& Petrides, 2012). The LPFC is organized in 6 cortical layers with a major expansion of layers $2 / 3$ where neurons encoding working memory have been identified (Arnsten, 2013; Constantinidis \& Wang, 2004). Neurons in this region have extensive functional connectivity with one another that depends on their receptive and memory field location (Leavitt, Pieper, Sachs, Joober, \& Martinez-Trujillo, 2013) and such fields cover the entire visual space (Bullock, Pieper, Sachs, \& Martinez-Trujillo, 2017).

One interesting question would be whether in vivo burst firing regimes in the HPC are mainly due to intrinsic properties of the principal cells. Indeed, studies in rodents have shown that CA3 neurons also fire bursts in vitro (Mizuseki et al., 2012; Ranck, 1973; Traub \& Wong, 1981). This bursting behaviour may be required to induce processes such as long-term potentiation (LTP) that shapes synaptic strength and enables coding of long-term memories (Bliss \& Collingridge, 1993). This suggests burst firing is robustly 'built up' into the intrinsic machinery of the HPC principal cell (e.g., membrane channel composition) rather than implemented into the HPC networks' dynamics. Such intrinsic cellular machinery would make the HPC a structure intrinsically built for burst firing that enables the processes underlying longterm memory formation (i.e., changes in synaptic strength that must occur effectively and rapidly in order to engrave memories into the network structure).

In the LPFC, a recent study has reported the existence of pyramidal cells that are intrinsically bursty based on their response profiles to square wave stimuli (González-Burgos et al., 2019). Interestingly, this study reported that burst neurons were more abundant in the LPFC relative to the lateral intraparietal area (LIP). Thus, it is possible that neocortical areas also differ in the way they cluster informative spikes in time depending on their involvement in long termmemory formation of their connectivity with the HPC and other areas. The latter may indicate that neural codes are heterogenous and can all serve information transmission, but at the same time they can serve different functions by changing the spatiotemporal features of spike trains. The features that make burst codes different across areas may be part of the intrinsic make up of individual cell types. The latter does not take away from the role of network connectivity and dynamics in shaping the neural code but can add heterogeneity and efficiency to neural communication and plasticity across the cortical mantle.

\section{Methods}

Four male rhesus macaques (Macaca mulatta) were used in these experiments, two in HPC experiments ( 7 and 14 years old, and $7 \mathrm{~kg}$ and $12 \mathrm{~kg}$ respectively) and two in LPFC experiments (10 and 9 years old, $12 \mathrm{~kg}$ and $10 \mathrm{~kg}$ respectively). They were trained on a context object association task in a virtual environment where they received juice rewards as positive reinforcement. All procedures followed Canadian Council on Animal Care guidelines and were carried out at either McGill University or Western University and were approved by the respective University Animal Care Committees.

Hippocampal recordings were carried out using 1-4 high impedance tungsten electrodes lowered each day to the right hippocampus, using co-registered image guidance for trajectory 
and depth, with examples seen in Figure 1e of recording locations. Most recordings were done in the mid to posterior putative CA3 region. Further information on electrode placement and targeting is available in Gulli et al. (2020). LPFC recordings were acquired using two 96-channel Utah arrays positioned at the posterior end of the principal sulcus, on the dorsal and ventral gyri of the principal sulcus and the anterior gyrus of the arcuate sulcus, spanning parts of area $8 a$ and of area $9 / 46$. The shank length was $1.5 \mathrm{~mm}$, and so was likely in layer II/III. Signals were acquired at $30 \mathrm{kHz}$ using one (HPC) or two (LPFC) 128-channel Cerebus recording systems (Blackrock Microsystems) and saved for later offline sorting, done with Plexon Offline sorter (version 4.5.0, Plexon Inc.). Time was not used as a feature during sorting, but units with waveform shapes that varied extensively or merged with other units were excluded from these analyses.

The tasks were very similar, taking place in a double ended $Y$ maze, termed the Xmaze and described previously (Doucet, Gulli, \& Martinez-Trujillo, 2016; Gulli et al., 2020). At the end of each $\mathrm{Y}$, were two colored discs towards one of which the animals would navigate to receive the associated reward. The reward was dictated by the context, which was indicated by a texture that was applied to the walls, either a dark grey "steel" texture or a brown "wood" one. The highest value color in one context was the lowest in the other context (Fig.1d). The LPFC recordings were done with only this high and low option, but the HPC had a middle color that was worth half the reward in both contexts. Monkeys used the joystick to navigate to their chosen colour, receive the associated reward, and then turn around and navigate back towards the other end to make another choice. Figure 1 shows an example trial trajectory, and the trajectories for two example sessions (Fig. 1b,c).

Behavioural Analysis

Monkeys were trained to be able to learn the task before recordings, and then presented with new combinations of colours each day, picked pseudo randomly to avoid a color occurring two days in a row. We used a performance analysis window of the 50 trials preceding the final 10 trials (excluded because performance may falter as satiation is reached).

Calculating burst propensity

The initial analysis of ISIs was just done by taking all spikes recorded during the task and pooling them for each area. We removed all ISIs greater than $60 \mathrm{~ms}$ and plotted the probability density function histogram of the population for each area. We calculated the burst fraction as the fraction of all ISIs during the task that were equal to or below $7 \mathrm{~ms}$. Because this could start to correlate with high firing rates, we also made a burst index for the neurons based on the ISI histogram and the predicted ISI distribution based on a Poisson distribution with the calculated firing rate. To predict the probability of ISIs of a certain duration, we followed the method of Livingstone et al. (Livingstone et al., 1996), taking the firing rate averaged over the whole task. Using equation 1 , where $\lambda=$ firing rate, and $t=$ time bin. We calculated the probability for each

$$
\text { eq. } 1 . \quad f(t)=\lambda e^{-\lambda t}
$$

$1 \mathrm{~ms}$ time bin from 2-40ms, and then normalized these measures by the sum of all these predictions. We then did the same thing with the measured ISIs, normalizing by the sum of the measured ISIs between 2 and $40 \mathrm{~ms}$. We then summed the predicted values from 2 to $7 \mathrm{~ms}$ and subtracted that from the sum of the measured values between 2 and $7 \mathrm{~ms}$. We divided this difference by the sum of the two sums to bind our index between -1 and 1 (see equation 2).

$$
\text { eq.2. } \quad \text { burst index }=\frac{\sum I S I s \text { measured }-\sum \text { ISIs predicted }}{\sum \text { ISIs measured }+\sum \text { ISIs predicted }}
$$

\section{Calculating information}

To calculate mutual information, we used only correct trials. Bursts were detected as any group of three or more spikes where all ISIs were equal or less than $7 \mathrm{~ms}$. The timing of the burst was the onset of the first spike, and which ever period the first spike occurred in was considered the period that the burst happened in. We split the task into four behaviourally separate periods: the 
post reward period, the context period, the decision period, and goal approach period. The post reward period started at the end of the reward administration and is potentially when the monkey is incorporating the knowledge gained from the previous trial. This continues until they navigate to the start of the corridor where the context appears, which is the start of the context period. The decision period starts at the appearance of the target objects, when they have to choose between the two, and the goal approach period starts at the beginning of the first turn of more than 10 degrees towards an object, and proceeds until they reach the target, just before reward administration. The period of reward administration was not analysed. The durations of each task period vary based on which period it is, and by trial, but we calculated rates based on the duration of each individual period. We used 60 sample rates from each trial period for each neuron to calculate the mutual information using the Neuroscience Information Theory Toolbox (Timme \& Lapish, 2018). We then ran 5000 shuffles to generate the $p$-value for the mutual information. We then ran this whole process, starting from the sampling, 50 times and analysed the means of the mutual information and the $p$-value for each neuron.

Decoding analysis

For the decoding analysis, we used support vector machine (SVM) decoding from LIBSVM v.3.23 using five-fold cross-validation and a linear kernel on rates that were normalized between 0 and 1 . We again randomly sampled 60 rate pairs from each trial period and ran the decoding analysis on each set of rates before shuffling the labels three times and decoding again to get three measures of chance decoding. We repeated this 100 times to take the mean accuracy and mean chance decoding of the same trials for both spike rate and burst rate decoding. To examine the differences between the performance of the burst and spike decoder within an area, and to compare this across areas, we created a decoding index (see equation 3 ). This gave us 100 index values to run a t-test on.

eq. 3. Spike-burst index $=\frac{\text { decoding accuracy for spike rate }- \text { decoding accuracy for burst rate }}{\text { decoding accuracy for spike rate }+ \text { decoding accuracy for burst rate }}$

\section{Optimized Decoders}

For the optimized ensembles, we used the same set up for the SVM, but for the pool of neurons we only used neurons that had significant mutual information $(p<.05)$ for a specific rate. This limited the number of neurons we could use. To control for differences in number of eligible neurons in the two areas, for an iteration of the optimized decoder, we would randomly select 30 neurons frow which we would build the decoder. For the first test, we used only neurons with significant mutual information for burst rate. Each neuron was tested individually to find the neuron with the best decoding accuracy. This neuron was then paired with every other neuron, and we ran an SVM on each duo to find the best duo. The best duo was used to find the best trio that included the best duo, and so on. This does not necessarily find the absolute optimal duo or trio, but it is an effective method for exploring the decoding space without having to exhaustively try all possible permutations, which can be computationally expensive. Indeed, it results in better decoding accuracies than simply using the best neurons based on individual performance, as illustrated in Leavitt et al (2017). After building the decoder to either 20 units for the initial analysis, or 18 units for the time window analysis (described below) we then selected another 30 random units and repeated the process to build another optimized decoder, building 50 optimized decoders in total to give us a population of results to analyse.

For the decoders built to assess the contribution of neurons we only used neurons with significant information in the burst rate. We fit an exponential function (eq. 4) to each performance curve, and calculated the point where performance was $95 \%$ of the asymptote which is defined as 1-c. This gave us 50 points for each set of decoders so we could run t-tests on these points within areas and within spike and burst rates.

$$
\text { eq. } 4 . \quad y=1-a * e^{-\left(\frac{x}{b}\right)}-c
$$


The time window analysis used different time windows within which to calculate the rates. Because we wanted to analyze the compression of the full signal, we only used the firing rates for this analysis, and used neurons that had significant firing rate mutual information values. We used time windows of $25,50,100,150,200,300,400$, and 500 milliseconds instead of integrating over the whole trial period. We chose to center the windows in the middle of the period. We built the optimized decoders with pools of 30 neurons, but only built them to 18 neurons because they would have already saturated decoding performance before then. We built 50 optimized decoders for each time window for each area. To fairly compare the effect of time windows, we took the maximum of the average performance across ensemble sizes of the best decoder $(500 \mathrm{~ms})$ and calculated the performance of all the decoders, at each ensemble size, as a fraction of such maximum performance. To compare these performance fractions, we took the $\mathrm{HC}$ fractions for each time window and sample size and subtracted the corresponding LPFC fractions, which gave us a population of differences at each ensemble size and time window. We ran a two-way ANOVA on these differences to assess for effects of time window size or ensemble size on the differences in performance. To determine which differences between the two areas were significantly different from 0 , we calculated the $95 \%$ confidence intervals for each ensemble size and time window and found those that did not include 0 . 


\section{References}

Arnsten, A. F. T. (2013). The Neurobiology of Thought: The Groundbreaking Discoveries of Patricia Goldman-Rakic 1937-2003. Cerebral Cortex, 23(10), 2269-2281. https://doi.org/10.1093/CERCOR/BHT195

Backen, T., Treue, S., \& Martinez-Trujillo, J. C. (2018). Encoding of Spatial Attention by Primate Prefrontal Cortex Neuronal Ensembles. ENeuro, 5(1), ENEURO.0372-16.2017. https://doi.org/10.1523/ENEURO.0372-16.2017

Bittner, K. C., Milstein, A. D., Grienberger, C., Romani, S., \& Magee, J. C. (2017). Behavioral time scale synaptic plasticity underlies CA1 place fields. Science, 357(6355), 1033-1036. https://doi.org/10.1126/science.aan3846

Bliss, T. V. P., \& Collingridge, G. L. (1993). A synaptic model of memory: Long-term potentiation in the hippocampus. Nature, 361(6407), 31-39. https://doi.org/10.1038/361031a0

Bullock, K. R., Pieper, F., Sachs, A. J., \& Martinez-Trujillo, J. C. (2017). Visual and presaccadic activity in area $8 \mathrm{Ar}$ of the macaque monkey lateral prefrontal cortex. Journal of Neurophysiology, 118(1), 15-28. https://doi.org/10.1152/jn.00278.2016

Buzsáki, G. (2015). Hippocampal sharp wave-ripple: A cognitive biomarker for episodic memory and planning. Hippocampus, 25(10), 1073-1188. https://doi.org/10.1002/hipo.22488

Constantinidis, C., \& Goldman-Rakic, P. S. (2002). Correlated discharges among putative pyramidal neurons and interneurons in the primate prefrontal cortex. Journal of Neurophysiology, 88(6), 3487-3497. https://doi.org/10.1152/jn.00188.2002

Constantinidis, C., \& Wang, X.-J. (2004). A neural circuit basis for spatial working memory. The Neuroscientist, 10(6), 553-565. https://doi.org/10.1177/1073858404268742

Doucet, G., Gulli, R. A., Corrigan, B. W., Duong, L. R., \& Martinez-Trujillo, J. C. (2020). Modulation of local field potentials and neuronal activity in primate hippocampus during saccades. Hippocampus, 30(3), 192-209. https://doi.org/10.1002/hipo.23140

Doucet, G., Gulli, R. a., \& Martinez-Trujillo, J. C. (2016). Cross-species 3D virtual reality toolbox for visual and cognitive experiments. Journal of Neuroscience Methods, 266, 8493. https://doi.org/10.1016/j.jneumeth.2016.03.009

Eichenbaum, H., Amaral, D. G., Buffalo, E. A., Buzsáki, G., Cohen, N., Davachi, L., ... Witter, M. (2016). Hippocampus at 25. Hippocampus, 26(10), 1238-1249. https://doi.org/10.1002/hipo.22616

Fuster, J. M., \& Alexander, G. E. (1971). Neuron Activity Related to Short-Term Memory. Science, 173(3997), 652-654. https://doi.org/10.1126/SCIENCE.173.3997.652

González-Burgos, G., Miyamae, T., Krimer, Y., Gulchina, Y., Pafundo, D. E., Krimer, O., ... Lewis, D. A. (2019). Distinct Properties of Layer 3 Pyramidal Neurons from Prefrontal and Parietal Areas of the Monkey Neocortex. Journal of Neuroscience, 39(37), 7277-7290. https://doi.org/10.1523/JNEUROSCI.1210-19.2019

Gulli, R. A., Duong, L. R., Corrigan, B. W., Doucet, G., Williams, S., Fusi, S., \& MartinezTrujillo, J. C. (2020). Context-dependent representations of objects and space in the primate hippocampus during virtual navigation. Nature Neuroscience, 23(1), 103-112. https://doi.org/10.1038/s41593-019-0548-3

Harris, K. D., Hirase, H., Leinekugel, X., Henze, D. A., \& Buzsáki, G. (2001). Temporal interaction between single spikes and complex spike bursts in hippocampal pyramidal cells. Neuron, 32(1), 141-149. https://doi.org/10.1016/S0896-6273(01)00447-0 
Kandel, E. R., Schwartz, J. H., Jessell, T. M., Siegelbaum, S. A., \& Hudspeth, A. J. (Eds.). (2012). Principles of Neural Science (5th ed.). McGraw-Hill.

Kepecs, A., Wang, X.-J., \& Lisman, J. (2002). Bursting Neurons Signal Input Slope. The Journal of Neuroscience, 22(20), 9053-9062. https://doi.org/10.1523/JNEUROSCI.22-2009053.2002

Leavitt, M. L., Pieper, F., Sachs, A. J., \& Martinez-Trujillo, J. C. (2017). Correlated variability modifies working memory fidelity in primate prefrontal neuronal ensembles. Proceedings of the National Academy of Sciences of the United States of America, 114(12), E2494E2503. https://doi.org/10.1073/pnas.1619949114

Leavitt, M. L., Pieper, F., Sachs, A., Joober, R., \& Martinez-Trujillo, J. C. (2013). Structure of spike count correlations reveals functional interactions between neurons in dorsolateral prefrontal cortex area 8a of behaving primates. PloS One, 8(4), e61503. https://doi.org/10.1371/journal.pone.0061503

Lisman, J. (1997). Bursts as a unit of neural information: making unreliable synapses reliable. Trends in Neurosciences, 20(1), 38-43. https://doi.org/10.1016/S0166-2236(96)10070-9

Livingstone, M. S., Freeman, D. C., \& Hubel, D. H. (1996). Visual responses in V1 of freely viewing monkeys. Cold Spring Harbor Symposia on Quantitative Biology, 61, 27-37. Retrieved from http://www.ncbi.nlm.nih.gov/pubmed/9246432

Mendoza-Halliday, D., \& Martinez-Trujillo, J. C. (2017). Neuronal population coding of perceived and memorized visual features in the lateral prefrontal cortex. Nature Communications 2017 8:1, 8(1), 1-13. https://doi.org/10.1038/ncomms15471

Mizuseki, K., Royer, S., Diba, K., \& Buzsáki, G. (2012). Activity dynamics and behavioral correlates of CA3 and CA1 hippocampal pyramidal neurons. Hippocampus, 22(8), 16591680. https://doi.org/10.1002/hipo.22002

O'Keefe, J., \& Nadel, L. (1978). The Hippocampus as a Cognitive Map. Oxford University Press.

Pernía-Andrade, A. J., \& Jonas, P. (2014). Theta-gamma-modulated synaptic currents in hippocampal granule cells in vivo define a mechanism for network oscillations. Neuron, 81(1), 140-152. https://doi.org/10.1016/j.neuron.2013.09.046

Ranck, J. B. (1973). Studies on single neurons in dorsal hippocampal formation and septum in unrestrained rats. Part I. Behavioral correlates and firing repertoires. Experimental Neurology, 41(2), 462-531. https://doi.org/10.1016/0014-4886(73)90290-2

Raus Balind, S., Magó, Á., Ahmadi, M., Kis, N., Varga-Németh, Z., Lőrincz, A., ... Makara, J. K. (2019). Diverse synaptic and dendritic mechanisms of complex spike burst generation in hippocampal CA3 pyramidal cells. Nature Communications, 10(1), 1-15. https://doi.org/10.1038/s41467-019-09767-w

Remy, S., \& Spruston, N. (2007). Dendritic spikes induce single-burst long-term potentiation. Proceedings of the National Academy of Sciences of the United States of America, 104(43), 17192-17197. https://doi.org/10.1073/pnas.0707919104

Roussy, M., Luna, R., Duong, L., Corrigan, B., Gulli, R. A., Nogueira, R., ... Martinez-Trujillo, J. C. (2021). Ketamine disrupts naturalistic coding of working memory in primate lateral prefrontal cortex networks. Molecular Psychiatry 2021, 1-16. https://doi.org/10.1038/s41380-021-01082-5

Simonnet, J., \& Brecht, M. (2019). Burst firing and spatial coding in subicular principal cells. Journal of Neuroscience, 39(19), 3651-3662. https://doi.org/10.1523/JNEUROSCI.165618.2019 
Skaggs, W. E., McNaughton, B. L., Permenter, M., Archibeque, M., Vogt, J., Amaral, D. G., \& Barnes, C. A. (2007). EEG Sharp Waves and Sparse Ensemble Unit Activity in the Macaque Hippocampus. Journal of Neurophysiology, 98(2), 898-910. https://doi.org/10.1152/jn.00401.2007

Spaak, E., Watanabe, K., Funahashi, S., \& Stokes, M. G. (2017). Stable and dynamic coding for working memory in primate prefrontal cortex. Journal of Neuroscience, 37(27), 6503-6516. https://doi.org/10.1523/JNEUROSCI.3364-16.2017

Thomas, M. J., Watabe, A. M., Moody, T. D., Makhinson, M., \& O’Dell, T. J. (1998). Postsynaptic complex spike bursting enables the induction of LTP by theta frequency synaptic stimulation. Journal of Neuroscience, 18(18), 7118-7126. https://doi.org/10.1523/jneurosci.18-18-07118.1998

Timme, N. M., \& Lapish, C. (2018). A tutorial for information theory in neuroscience. ENeuro, 5(3). https://doi.org/10.1523/ENEURO.0052-18.2018

Traub, R. D., \& Wong, R. K. S. (1981). Penicillin-induced epileptiform activity in the hippocampal slice: A model of synchronization of CA3 pyramidal cell bursting. Neuroscience, 6(2), 223-230. https://doi.org/10.1016/0306-4522(81)90058-0

Tremblay, S., Pieper, F., Sachs, A., \& Martinez-Trujillo, J. (2015). Attentional Filtering of Visual Information by Neuronal Ensembles in the Primate Lateral Prefrontal Cortex. Neuron, 85(1), 202-215. https://doi.org/10.1016/j.neuron.2014.11.021

Voloh, B., \& Womelsdorf, T. (2018). Cell-type specific burst firing interacts with theta and beta activity in prefrontal cortex during attention states. Cerebral Cortex, 28(12), 4348-4364. https://doi.org/10.1093/cercor/bhx287

Womelsdorf, T., Ardid, S., Everling, S., \& Valiante, T. A. (2014). Burst firing synchronizes prefrontal and anterior cingulate cortex during attentional control. Current Biology, 24(22), 2613-2621. https://doi.org/10.1016/j.cub.2014.09.046

Xu, W., Morishita, W., Buckmaster, P. S., Pang, Z. P., Malenka, R. C., \& Südhof, T. C. (2012). Distinct Neuronal Coding Schemes in Memory Revealed by Selective Erasure of Fast Synchronous Synaptic Transmission. Neuron, 73(5), 990-1001. https://doi.org/10.1016/j.neuron.2011.12.036

Yeterian, E. H., Pandya, D. N., Tomaiuolo, F., \& Petrides, M. (2012). The cortical connectivity of the prefrontal cortex in the monkey brain. Cortex, 48(1), 58-81. https://doi.org/10.1016/J.CORTEX.2011.03.004

Zeldenrust, F., Wadman, W. J., \& Englitz, B. (2018). Neural coding with bursts-current state and future perspectives. Frontiers in Computational Neuroscience, 12, 48. https://doi.org/10.3389/fncom.2018.00048 
Supplementary Figures

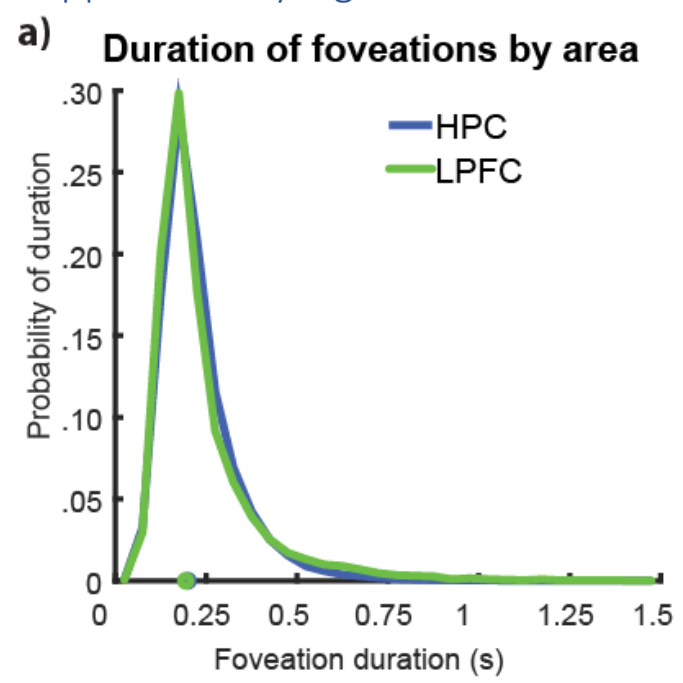

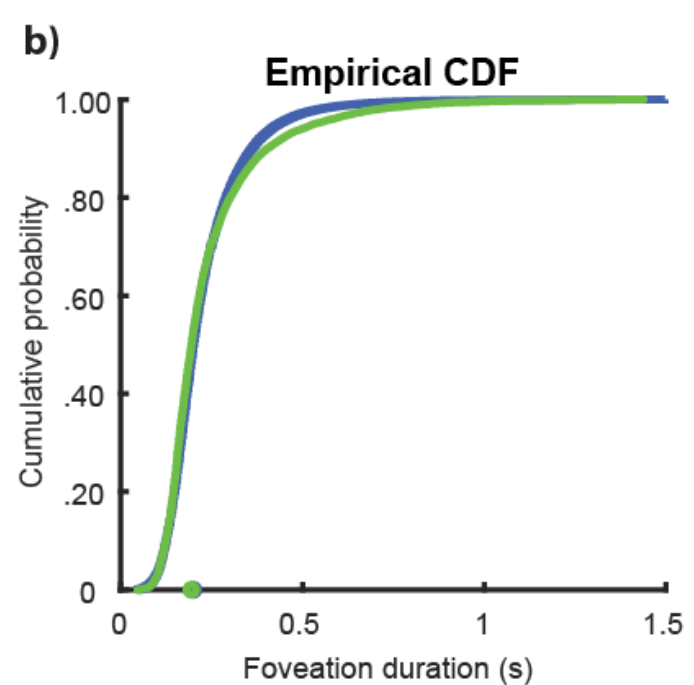

Supplementary Figure 1. a) the duration of foveations, or intersaccadic intervals for the NHPs used in either the HPC or LPFC recordings show a very similar distribution. b) The Empirical Cumulative Density Function of the foveation durations show there is very high overlap, with a very low Kolmogorov-Smirnov D of .05, which does reach significance due to the high power of this analysis with many thousands of foveations across many sessions. 